\title{
In-depth mutational analyses of colorectal neuroendocrine carcinomas with adenoma or adenocarcinoma components
}

\author{
Christine Woischke ${ }^{1,12}$, Christian W Schaaf ${ }^{1,12}$, Hui-Min Yang ${ }^{2,3}$, Michael Vieth ${ }^{4}$, \\ Lothar Veits ${ }^{4}$, Helene Geddert ${ }^{5}$, Bruno Märkl ${ }^{6}$, Peter Stömmer ${ }^{7}$, David F Schaeffer ${ }^{2}$, \\ Matthias Frölich ${ }^{1}$. Helmut Blum ${ }^{8,9,10}$, Sebastian Vosberg $9,10,11$, Philipp A Greif ${ }^{9,10,11}$, \\ Andreas Jung ${ }^{1,9,10}$, Thomas Kirchner ${ }^{1,9,10}$ and David Horst ${ }^{1,9,10}$ \\ ${ }^{1}$ Pathologisches Institut der Ludwig-Maximilians-Universität (LMU), München, Germany; ${ }^{2}$ Department of \\ Pathology and Laboratory Medicine, The University of British Columbia, Vancouver, BC, Canada; \\ ${ }^{3}$ Department of Pathology and Cell Biology, Columbia University, New York, NY, USA; ${ }^{4}$ Institut für \\ Pathologie, Klinikum Bayreuth, Bayreuth, Germany; ${ }^{5}$ Institut für Pathologie, St Vincentius-Kliniken, \\ Karlsruhe, Germany; ${ }^{6}$ Institut für Pathologie, Klinikum Augsburg, Augsburg, Germany; ${ }^{7}$ Pathologicum \\ Augsburg, Augsburg, Germany; ${ }^{8}$ Laboratory for Functional Genome Analysis (LAFUGA), at the Gene Center, \\ Ludwig-Maximilians-Universität (LMU), München, Germany; ${ }^{9}$ German Cancer Consortium (DKTK), \\ Heidelberg, Germany; ${ }^{10}$ German Cancer Research Center (DKFZ), Heidelberg, Germany and ${ }^{11}$ Department of \\ Internal Medicine 3, University Hospital, Ludwig-Maximilians-Universität (LMU), München, Germany
}

\begin{abstract}
Neuroendocrine carcinomas (NECs) of the colorectum are rare but highly aggressive neoplasms. These tumors show some shared genetic alterations with colorectal adenocarcinomas, and most of them have adjacent glandular adenoma or adenocarcinoma components. However, genetic data on colorectal NECs still are sparse and insufficient for definite conclusions regarding their molecular origin. Based on morphological characterization, panel and whole-exome sequencing, we here present results from an in-depth analysis of a collection of 15 colorectal NECs with glandular components, 10 of which by definition were mixed adenoneuroendocrine carcinomas (MANECs). Among shared genetic alterations of both tumor components, we most frequently found TP53, KRAS and APC mutations that also had highest allele frequencies. Mutations exclusive to glandular or neuroendocrine components outnumbered shared mutations but occurred at lower allele frequencies. Our findings not only provide additional evidence for a common clonal origin of colorectal NECs and adjacent glandular tumor components, but strongly suggest their development through the classical adenoma-carcinoma sequence. Moreover, our data imply early separation of glandular and neuroendocrine components during malignant transformation with subsequent independent mutational evolution.
\end{abstract}

Modern Pathology (2017) 30, 95-103; doi:10.1038/modpathol.2016.150; published online 2 September 2016

Neuroendocrine carcinomas (NECs) are biologically aggressive cancers with poor prognosis. In the colorectum, they are observed at low frequencies of $0.1-0.6 \% .^{1,2}$ NECs are defined by the expression of markers indicative of neuroendocrine differentiation, such as synaptophysin, chromogranin $\mathrm{A}$ and CD56, and show high proliferation rates that

Correspondence: Professor D Horst, MD, Pathologisches Institut der Ludwig-Maximilians-Universität (LMU), Thalkirchner Str. 36, München 80337, Germany.

E-mail: david.horst@med.uni-muenchen.de

${ }^{12}$ These authors contributed equally to this work.

Received 11 April 2016; revised 17 July 2016; accepted 18 July 2016; published online 2 September 2016 distinguish them from less aggressive neuroendocrine tumors (NETs). Based on tumor cell size and histologic appearance, small and large cell NECs can be distinguished from one another. ${ }^{3}$

Interestingly, NECs and NETs of the colorectum appear biologically unrelated. The genetic background of colorectal NECs includes loss of heterozygosity of $A P C$, TP53 and DCC genes as well as mutations in TP53, KRAS and BRAF genes, found in typical adenocarcinomas at this site. ${ }^{4-8}$ In addition, $61 \%$ of large cell NECs and $26 \%$ of small cell NECs of the colorectum are accompanied by adjacent glandular adenoma or adenocarcinoma components, and these tumors are defined as mixed adenoneuroendocrine 
Table 1 Case characteristics

\begin{tabular}{|c|c|c|c|c|c|}
\hline Case & $\begin{array}{c}\text { Age } \\
\text { (years) }\end{array}$ & Sex & Localization & Glandular component histology & $\begin{array}{l}\text { Neuroendocrine carcinoma } \\
\text { histology }\end{array}$ \\
\hline 1 & 91 & $\mathrm{~F}$ & Right colon & Tubulovillous adenoma & Small cell, trabecular \\
\hline 2 & 82 & M & Right colon & Tubulovillous adenoma & Large cell, trabecular \\
\hline 3 & 72 & M & Right colon & Tubulovillous adenoma & Large cell, rosette \\
\hline 4 & 74 & M & Rectosigmoid & Tubulovillous adenoma & Large cell, trabecular, rosette \\
\hline 5 & 73 & M & Rectum & Tubulovillous adenoma & Small cell, nested \\
\hline 6 & 68 & $\mathrm{M}$ & Right colon & $\begin{array}{l}\text { Tubulovillous adenoma, low-grade partially mucinous } \\
\text { adenocarcinoma }\end{array}$ & Small cell, solid, trabecular \\
\hline 7 & 76 & $\mathrm{~F}$ & Right colon & Traditional serrated adenoma, low-grade adenocarcinoma & Large cell, solid, trabecular, rosette \\
\hline 8 & 58 & $\mathrm{~F}$ & Rectum & High-grade adenocarcinoma & Large cell, trabecular \\
\hline 9 & 72 & M & Sigmoid & Low-grade adenocarcinoma & Large cell, rosette \\
\hline 10 & 64 & M & Right colon & $\begin{array}{l}\text { Traditional serrated adenoma, high-grade mucinous } \\
\text { adenocarcinoma }\end{array}$ & Large cell, solid \\
\hline 11 & 61 & M & Rectum & Low-grade adenocarcinoma & Large cell, nested \\
\hline 12 & 77 & M & Rectum & Low-grade adenocarcinoma & Small cell, trabecular \\
\hline 13 & 71 & M & Right colon & Low-grade adenocarcinoma & Large cell, trabecular \\
\hline 14 & 52 & $\mathrm{~F}$ & Right colon & Low-grade partially mucinous adenocarcinoma & Large cell, trabecular \\
\hline 15 & 65 & $\mathrm{~F}$ & Rectum & Low-grade adenocarcinoma & Large cell, rosette, cribriform \\
\hline
\end{tabular}

carcinomas (MANECs) by the WHO, if adenocarcinoma and NEC components both exceed $30 \%$ of the neoplasm. ${ }^{3,9}$ In contrast, the less aggressive NETs usually lack such glandular components and show genetic alterations, which are distinct from those in NECs. ${ }^{7,8,10}$ Therefore, the molecular carcinogenesis of colorectal NECs appears to be closer related to typical colorectal adenocarcinomas than to NETs.

Colorectal NECs with adjacent glandular tumor components may provide an ideal model to study their genetic basis. Few previous studies on MANECs have identified overlapping genetic characteristics of glandular and neuroendocrine components and therefore suggested a common clonal origin. ${ }^{8,11-13}$ In addition, analyses of allelic imbalances in a few MANECs even suggested clonal evolution of NECs from adenocarcinomas. ${ }^{14}$ However, the methodologies applied and the small number of cases were insufficient for definite conclusions regarding the molecular origin of these tumors.

Here, we present results from an in-depth phenotypic and genetic analysis of a collection of 15 colorectal NECs with adjacent glandular components. Based on next-generation panel and wholeexome sequencing, our data demonstrate that such NECs not only share genetic alterations with their glandular components but also show typical genetic founder mutations of the classical colorectal adenoma-carcinoma sequence. They originate from this sequence by clonal evolution and early glandular and neuroendocrine component separation.

\section{Materials and methods}

\section{Tumor Specimens, Histology and Immunohistochemistry}

Archival tissue of 15 formalin-fixed and paraffinembedded cases of colorectal NECs with glandular components were retrieved from the Institutes of Pathology in Munich, Bayreuth, Karlsruhe, Augsburg and Vancouver (BC, Canada). The tumors were biopsied or resected between 2007 and 2013. All cases were re-evaluated based on the 2010 WHO classification and histologically fulfilled the diagnostic criteria of NECs or MANECs. ${ }^{3}$ Case characteristics are given in Table 1. Samples were anonymized and the study was approved by the local ethics committees. For histologic preparation, $5 \mu \mathrm{m}$ sections were deparaffinized and stained with hematoxylin and eosin (H\&E). For immunohistochemistry, sections were incubated with prediluted rabbit anti-CD56 (MRQ42), rabbit anti-synaptophysin (MRQ-40), mouse anti-chromogranin A (LK2H10), mouse anti-neuron-specific enolase (NSE; MRQ-55, Ventana Medical Systems) or mouse anti-Ki67 antibody (MIB-1, 1:150, Dako). Staining was done on a Ventana Benchmark XT autostainer and developed with ultraView Universal DAB detection kits (Ventana Medical Systems).

\section{Tumor Cell Quantification and DNA Extraction}

We used sections stained for H\&E, CD56, synaptophysin and chromogranin A to delineate pure glandular and NEC components. Tumor cellularity was then determined by counting tumor and nontumor cells in these areas microscopically. These sections then were used as blueprints to separately isolate adenocarcinoma and/or adenoma and NEC components under microscopic control from deparaffinized serial sections with sterile scalpel blades. Tumor DNA then was extracted with QIAamp DNA Micro Kits and GeneRead DNA FFPE Kits (Qiagen) for subsequent analyses of KRAS gene mutations, panel and exome sequencing, respectively. 


\section{Pyrosequencing}

The mutational status of KRAS exon 2 was analyzed by pyrosequencing on a PyroMark Q24 Advanced instrument (Qiagen) with primers 5'-NNNGG CCTGCTGAAAATGACTGAA-3' and biotin-5'-TTA GCTGTATCGTCAAGGCACTCT-3' for amplification, and 5'-TGTGGTAGTTGGAGCT-3' for sequencing, as previously described. ${ }^{15}$

\section{Panel Sequencing}

For next-generation panel sequencing, we used the Ion AmpliSeq Cancer Hotspot Panel v2, covering the mutational status of 50 oncogenes and tumorsuppressor genes (Life Technologies), according to the manufacturer's protocol. In brief, we amplified a total of 1-10 ng of Qubit quantified DNA with primer pools by PCR, and constructed libraries with Ion AmpliSeq Library Kits and Ion Xpress Barcode Adapters (Thermo Fisher). After emulsion PCR and bead purification, multiplexed libraries then were loaded onto 318 chips, and sequenced on an Ion Personal Genome Machine (all Thermo Fisher). For data analysis, sequence reads were mapped to human reference genome hg19 and filtered for nonsynonymous variants using Ion reporter software v5.0 (Thermo fisher). Annotations, information on pathogenesis and allele frequencies were retrieved from Ensembl VEP (www.ensembl.org/Homo_ sapiens/Tools/VEP).

\section{Whole-Exome Sequencing}

For exome sequencing, we fragmented 1-3 $\mu \mathrm{g}$ of genomic DNA, extracted from normal mucosa, glandular and neuroendocrine components of three cases, to an average size of $150 \mathrm{bp}$ with a Bioruptor sonicator (Diagenode). Paired-end sequencing libraries then were prepared using a DNA sample prep reagent set 1 (NEBNext). Library preparation included end repair, adapter ligation and PCR enrichment, and was carried out as recommended by Illumina protocols. Protein-coding sequences were captured using SureSelect Human All Exon $50 \mathrm{Mb}$ kits version 3 (Agilent) according to the manufacturer's instructions. Paired-end sequencing on a Genome Analyzer IIx platform (Illumina) resulted in 2x76-bp sequence reads. Base calling was done by the Illumina pipeline with default settings. Copy number aberrations were detected in exome data sets as described previously. ${ }^{16}$ Using Biomedical Genomics Workbench v2.5.4 (Qiagen), tumor sequencing reads of glandular and NEC components then were mapped to each other and to reads of normal colonic mucosa. Reads then were filtered for the Homo sapiens Ensembl v74 mRNA target region with a minimum coverage of 30x. Nonsynonymous variants, germline variants, variants outside the target region and variants annotated as
SNPs in the 1000 genomes database were removed. Finally, variants with allele frequencies $\geq 10 \%$ and a coverage $\geq 20 \mathrm{x}$ were filtered for those annotated in COSMIC (cancer.sanger.ac.uk/cosmic).

\section{Results}

\section{Clinical and Pathologic Characteristics of Colorectal NECs with Glandular Components}

We initially characterized our collection of 15 colorectal NECs with glandular components, 10 of which fulfilled the diagnostic criteria of MANECs, ${ }^{3}$ on the clinical and morphological level (Table 1). Two-thirds of the patients were males and one-third females. The average age at diagnosis was 70 years (range 52-91 years) and thus similar to the average age of patients diagnosed with colorectal adenocarcinomas. ${ }^{17}$ Tumors were distributed throughout the colon with $53 \%$ in the right hemicolon and $47 \%$ in the left hemicolon or rectum. Glandular exocrine tumor components were typical tubulovillous colorectal adenomas with low-grade dysplasia (5 cases, 33\%; Figure 1, left panels), classical low-grade colorectal adenocarcinomas (8 cases, 53\%; Figure 1, right panels), two of which were partially mucinous, or high-grade colorectal adenocarcinomas (2 cases, $13 \%$ ), one of which was mucinous. Of note, three adenocarcinomas had additional adjacent adenoma components, one of which was tubulovillous and two of which were traditional serrated adenomas (Table 1, Supplementary Figure 1). In all cases, these glandular tumors abruptly transitioned into high-grade NECs that either had a small cell $(27 \%)$ or large cell $(73 \%)$ phenotype (Figure 1). Here, the most frequent growth pattern was trabecular (60\%), and we also commonly observed rosette formations (33\%). In NEC components, neuroendocrine differentiation was confirmed by analyzing chromogranin A, CD56 antigen and synaptophysin that were expressed in $87 \%, 60 \%$ and $80 \%$, respectively (Figure $2 \mathrm{a}$ ). In case 6 , where only CD56 expression was noted, neuroendocrine differentiation was further confirmed by strong positivity for NSE (data not shown). We then analyzed the proliferation rate and found an average Ki67-index of $71.4 \%$ in the NEC components, which significantly exceeded that of $49.0 \%$ in the glandular components $(P<0.01$ by $t$-test, Figure $2 \mathrm{~b})$. Differences in proliferation rate between small and large cell NECs were not significant (data not shown). In summary, these cases were tumors of elderly patients and comprised a spectrum of adenomas and low- or high-grade colorectal adenocarcinomas with abrupt transition to highly proliferative small or large cell NECs.

\section{Identical KRAS Mutations Suggest a Clonal Origin of Glandular and NEC Components}

To obtain initial insights on whether NECs and glandular tumor components were clonally related, 

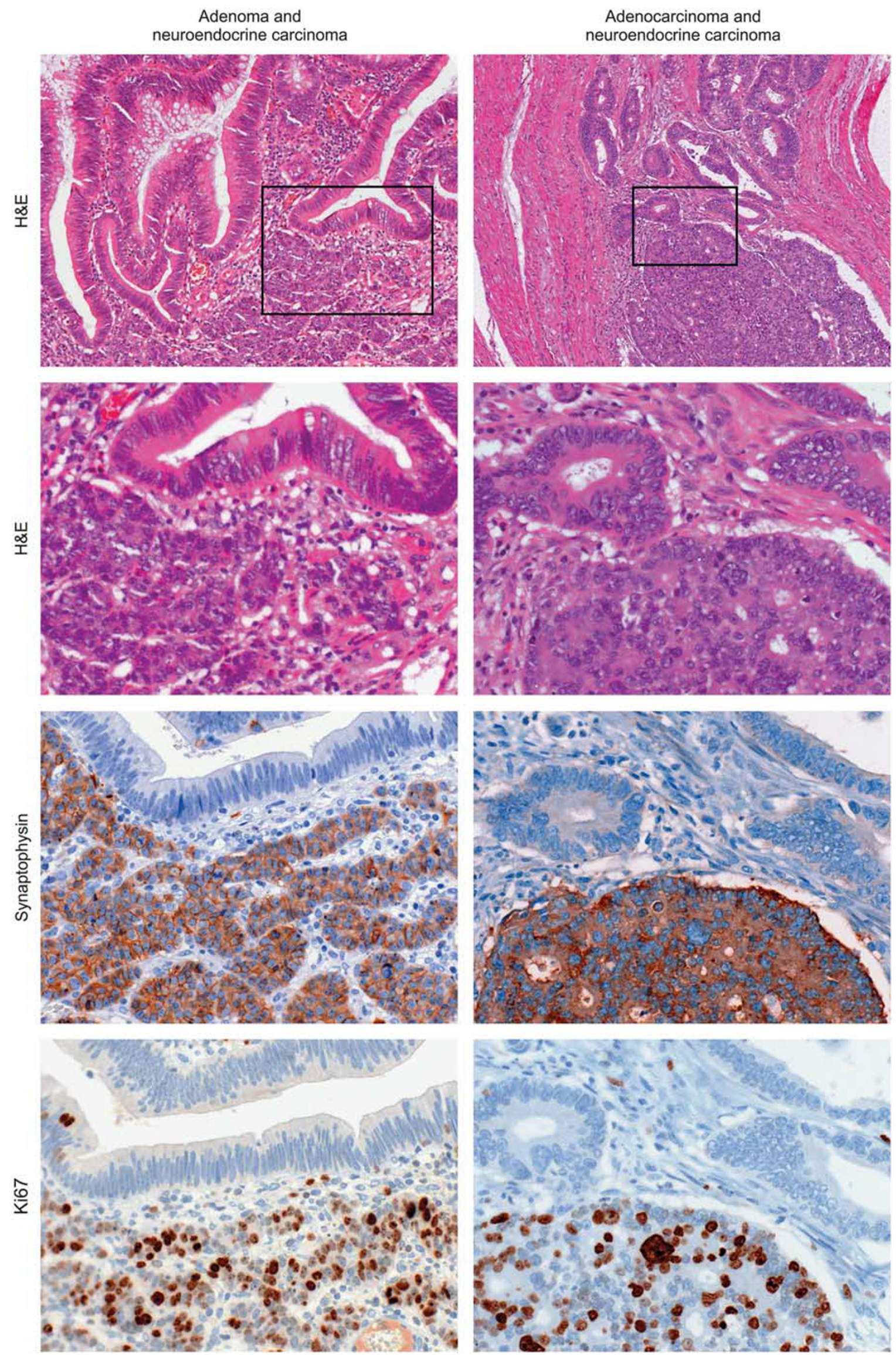
we segregated them during microdissection and analyzed them for KRAS gene mutations. Nine cases $(60 \%)$ had mutations in codons 12 or 13 of KRAS exon 2 , most frequently G13D (20\%). The remaining six cases (40\%) were wild-type for KRAS (Figure 2c). Importantly, for all cases, the KRAS mutational status was identical in glandular and neuroendocrine components $\left(P<10^{-14}\right.$ by kappa statistics). Based on KRAS mutational status alone, these findings strongly suggested a common clonal origin of small or large cell NECs and adjacent glandular components in the colorectum.

\section{Panel Sequencing Confirms Clonal Origin and Reveals Typical Mutations in Colorectal NECs with Glandular Components}

For further insights into the mutational spectrum, we subjected both glandular and neuroendocrine components of 10 cases to next-generation sequencing. At an average coverage of $2760 x$, we detected a total of 130 mutations, 55 and 75 of which were in glandular and neuroendocrine components, respectively (Supplementary Table 1). Examining overall mutation frequencies, $32.6 \%$ of mutations were shared among glandular and NEC components, whereas $40.7 \%$ occurred exclusively in neuroendocrine components, and $26.7 \%$ occurred only in the glandular components. Mutations were most frequently found in TP53, KRAS and APC genes, with $62 \%$ of these mutations shared between both components (Figures 3a and b), irrespective of the morphology of the neuroendocrine component. In frequency, these were followed by PIK3CA mutations, which occurred more often in the glandular components, and by $R B 1$ and MET mutations, both slightly more common in neuroendocrine components, although these differences were not significant (Figure 3b). Of note, when we separately analyzed adenomas that were-as a third tumor component-adjacent to adenocarcinomas in three cases, we found that TP53, APC and KRAS mutations were shared among adenoma and adenocarcinoma components (data not shown). Although these findings strongly substantiated clonal origins of NECs and glandular components, they also demonstrated independent additional mutational evolution of both components after clonal separation.

Next, to identify mutations that potentially were acquired early in malignant transformation, and thus define possible founding clones of NECs, we analyzed mutant allele frequencies. By tumor cell quantification, we determined a mean tumor cellularity of $82.2 \%$ (Supplementary Table 1). Mutations in founding clones would thus be expected at frequencies of $41.1 \% \quad( \pm 2$ s.d.), whereas higher allele frequencies may in addition indicate loss of heterozygosity. ${ }^{18,19}$ Within these criteria, mostly mutations in TP53, KRAS and APC, as well as individual mutations in $R B 1, M E T, B R A F, E R B B 4$ and PTPN11 qualified as potential founding clone mutations (Figure 3c). On the contrary, mutations in other genes, including PIK3CA, had lower allele frequencies and thus were assumed to be subclonal. Interestingly, shared mutations not only occurred at overall higher allele frequencies but also more frequently qualified as potential founding clone mutations when compared with mutations that were exclusive to either the glandular or neuroendocrine components $(P<0.001$ by chi-square test, Figure $3 \mathrm{~d})$. Of note, similar results were obtained, when cases with small and large cell NEC morphology were analyzed separately (Supplementary Figure 2). Although these data provided further evidence for a common clonal progenitor of both the glandular and neuroendocrine components with early separation and subsequent independent subclonal evolution, they also suggested presence of typical key mutations of colorectal adenocarcinomas in founding clones of NECs.
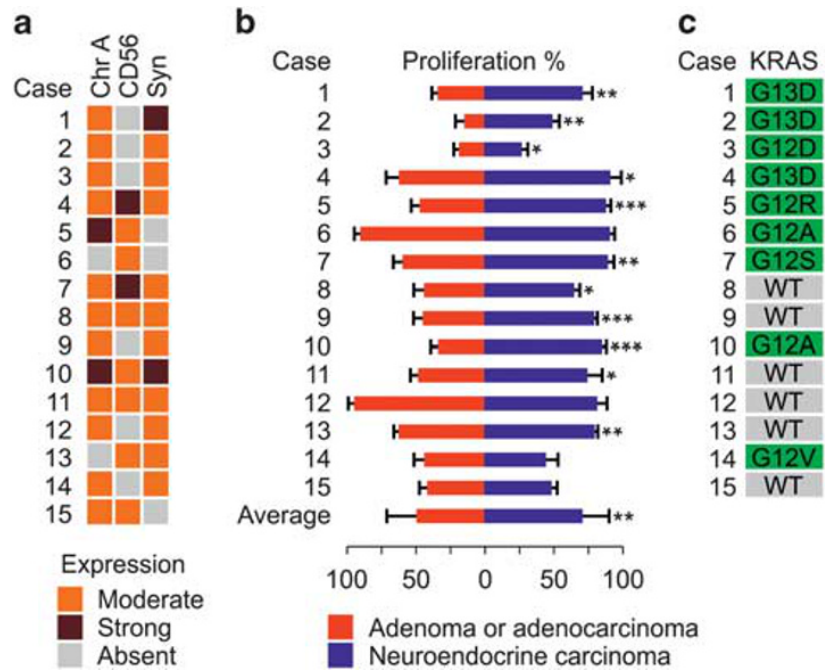

Figure 2 Phenotype and KRAS mutations in colorectal neuroendocrine carcinomas (NECs) with glandular components. (a) Strength of protein expression as determined by immunohistochemistry for chromogranin A (Chr A), CD56 and synaptophysin (Syn) in NEC components. (b) Quantification of proliferation, as assessed by Ki67 immunostaining, for glandular adenoma or adenocarcinoma and NEC tumor components. ${ }^{*} P<0.05$, ${ }^{* *} P<0.01,{ }^{* * *} P<0.001$ by $t$-test. (c) Glandular and neuroendocrine components were separately analyzed for mutations in KRAS exon 2. In each case, the indicated mutation or wild-type (WT) was found in both tumor components.

Figure 1 Morphological characteristics of colorectal neuroendocrine carcinomas (NECs) with glandular components. Tumors either contained colonic adenomas (left panels) or adenocarcinomas (right panels) that abruptly transitioned to NECs. Examples of neuroendocrine differentiation and proliferation are shown by immunostaining for synaptophysin and Ki67, respectively. 


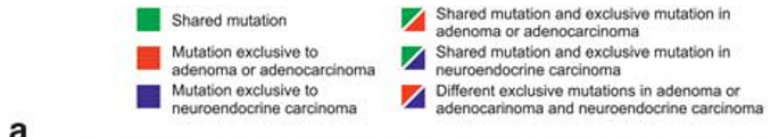

a
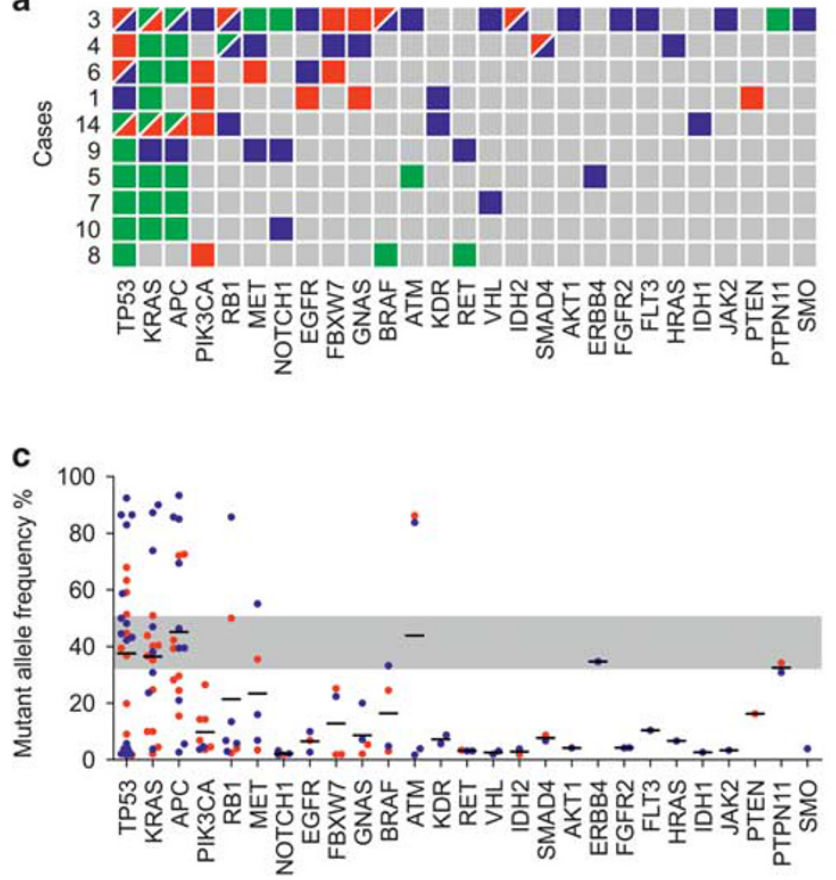

b
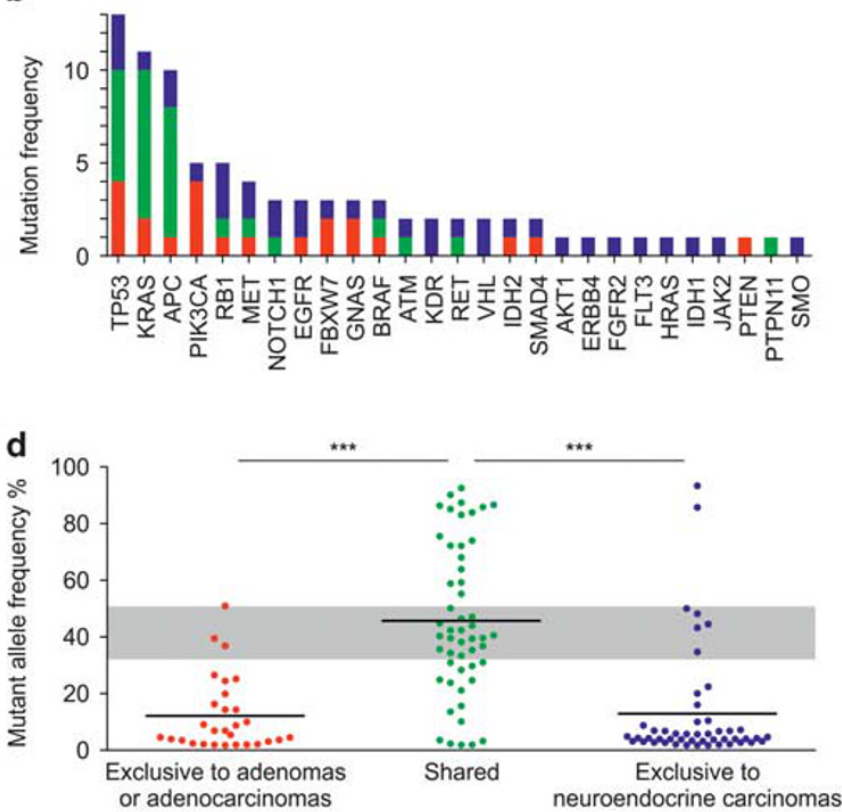

Figure 3 Mutational landscape of colorectal neuroendocrine carcinomas (NECs) with glandular components in panel sequencing. (a) Distribution of shared and exclusive non-synonymous mutations as indicated by colored cells. Rows represent cases and columns represent genes. (b) Absolute mutation frequencies by gene. Colors indicate shared mutations (green), and mutations exclusive to glandular (red) or neuroendorcrine (blue) components. (c) Allele frequencies of mutations by gene. Dots indicate mutations found in glandular (red dots) or neuroendocrine (blue dots) tumor components. (d) Allele frequencies of shared and exclusive mutations. ${ }^{* * *} P<10^{-8}$ by $t$-test. In (c, d), horizontal lines indicate means, and gray shaded zones indicate the expected range of mutant allele frequencies in founding clones. Mutant allele frequencies above indicate possible loss of heterozygosity.

\section{Exome Sequencing Reveals Distinct Degrees of Mutational and Chromosomal Overlap in NECs and Glandular Components}

To more broadly define similarities and differences of glandular and neuroendocrine components, we subjected three cases to whole-exome sequencing. With $83.5 \%$ of the target region covered by at least 20 reads, we were able to detect an average of 113 mutations per tumor component with a minimum coverage of 20x (Supplementary Table 2). These mutations included $44 \%$ of those found by panel sequencing. Importantly, on a broad level, exome sequencing confirmed the presence of shared mutations $(n=56,9.0 \%)$ in all three cases but also revealed that most mutations were either exclusive to glandular $(n=277,44.4 \%)$ or neuroendocrine $(n=291,46.6 \%)$ components (Figure 4a). Similar to our findings from panel sequencing, shared mutations had significantly higher allele frequencies than mutations that were exclusive to glandular or neuroendocrine components (Figure 4b). We then analyzed somatic copy number aberrations based on exome data sets, and discovered that, despite shared mutations, case 6 exhibited completely different copy number aberrations in glandular and neuroendocrine components $(r=-0.13$, Pearson correlation), whereas case $7(r=0.61)$ and case 9 $(r=0.88)$ showed similar aberrations (Figure 4c). Interestingly, when looking at mutations of these cases (Figure 3a), distinct chromosomal copy number aberrations coincided with distinct TP53 mutations in case 6, suggesting that chromosomal instability through loss of p53 function occurred after glandular and neuroendocrine component separation. On the contrary, similar chromosomal copy number aberrations coincided with shared TP53 mutations in both components of cases 7 and 9, suggesting a common clonal progenitor with stabilized chromosomal aberrations before component separation. Collectively, these data implied early clonal divergence and independent evolution of colorectal NECs and glandular components from a common clonal origin. However, distinct chromosomal alterations in these tumor components may still be observed.

\section{Discussion}

Here, we report our findings on a relatively large collection of colorectal NECs with glandular 
a
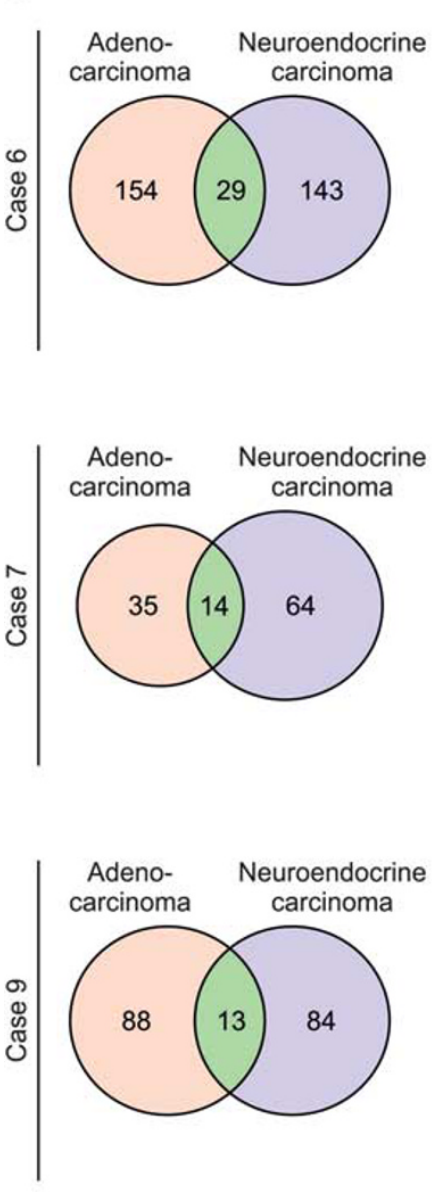

b
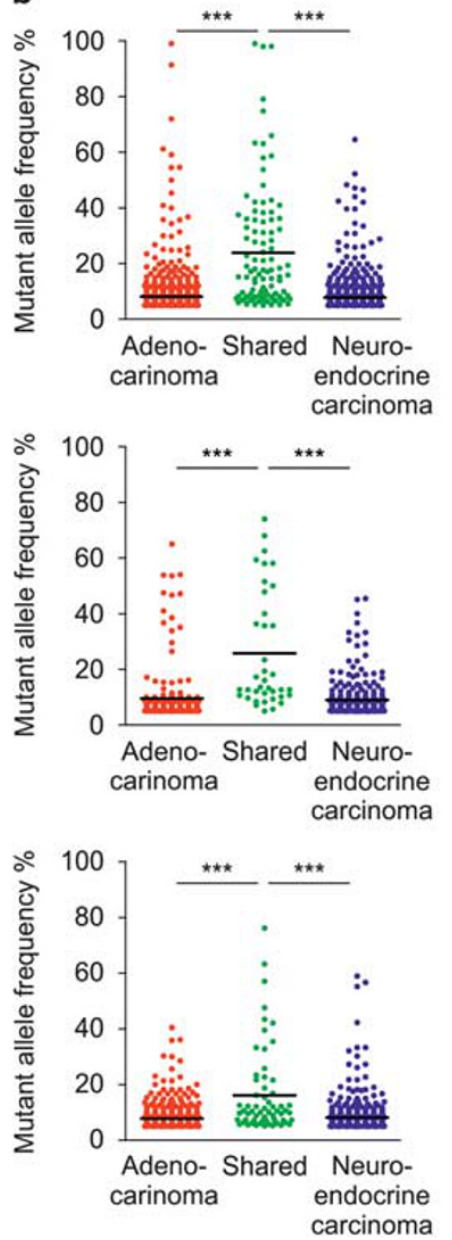
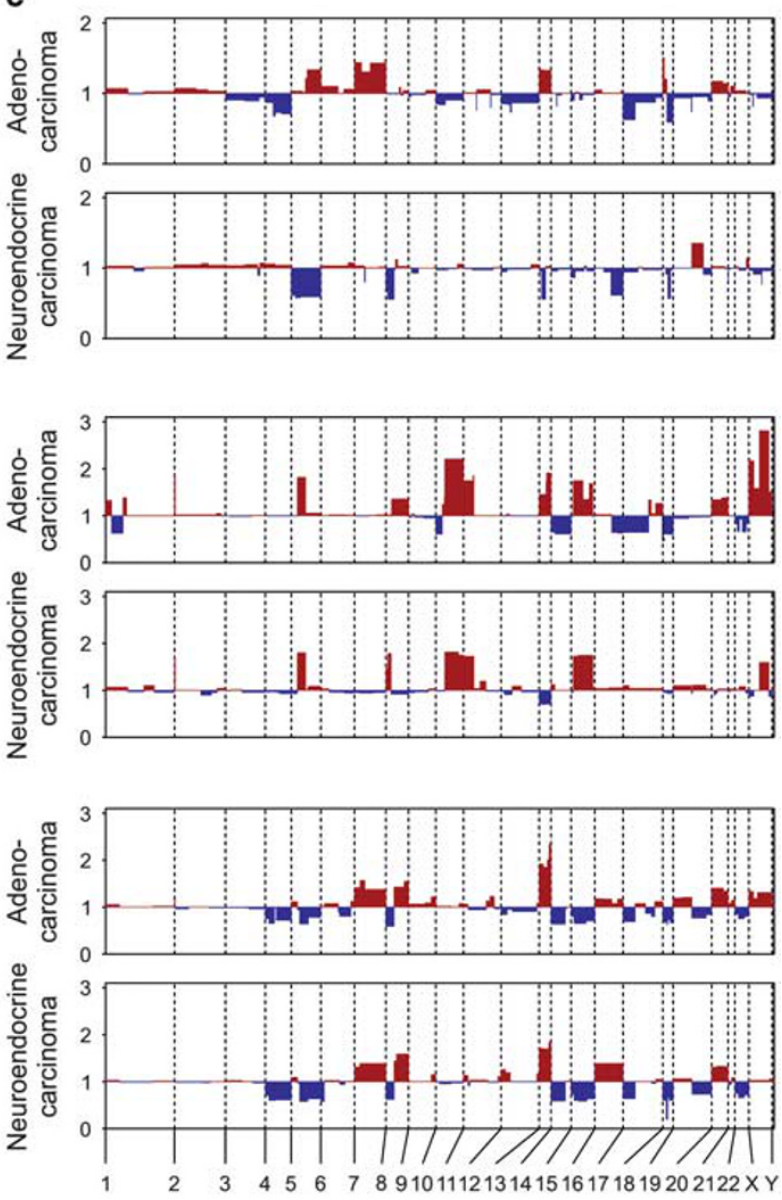

Chromosome position

Figure 4 Mutation frequencies and copy number aberrations of colorectal neuroendocrine carcinomas with glandular components found by whole-exome sequencing. (a) Frequencies of exclusive and shared mutations as indicated by Venn diagrams. (b) Allele frequencies of shared and exclusive mutations. Horizontal lines indicate means. ${ }^{* *} P<0.0001$ by $t$-test. (c) Ratios of frequencies of somatic copy number gains (red) and losses (blue) along the exome with indicated chromosomal positions.

components, including cases that qualify for the definition of colorectal MANECs. ${ }^{3}$ We demonstrate that the glandular components of these tumors are typical colorectal adenomas or adenocarcinomas that abruptly transition into highly proliferative NECs, mimicking collision tumors, as previously described. ${ }^{20}$ Despite the tumor collision hypothesis, several previous studies on similar individual cases or case series had reported shared genetic alterations-such as loss of heterozygosity or mutations-and therefore suggested a common clonal origin of both glandular and neuroendocrine components in these tumors. ${ }^{8,11-13}$ Our findings of identical KRAS mutation status, as well as several additional shared mutations that we identified by panel and exome sequencing in both components further support this idea, and indicate development of these tumors from a common clonal progenitor lesion or founding clone at high confidence levels.

However, we also demonstrate that both components had acquired exclusive mutations that even outnumbered shared mutations in our whole-exome sequencing data. This sheds additional light on the development of these tumors, as it implied that separation of NECs and their glandular components from a common progenitor clone occurred early during tumor development. Additional exclusive mutations then were acquired independently by both components. This idea of early separation is further supported by our finding that exclusive mutations showed lower allele frequencies and thus were mostly subclonal, when compared with shared mutations of putative common progenitor or founding clones. In this context, exome sequencing also revealed that distinct chromosomal aberrations may be observed in cases with distinct TP53 mutations, despite an otherwise common mutational genetic basis of both tumor components. Therefore, distinct allelic imbalances of glandular and neuroendocrine components, as reported for few MANECs, do not necessarily imply a distinct clonal origin. ${ }^{21}$

Among genes most frequently mutated in colorectal small and large cell NECs with glandular components, we identified TP53, KRAS and APC. These three 
genes are also most frequently mutated in typical colorectal adenocarcinomas and deregulate p53, MAPK and WNT signaling pathways. ${ }^{22}$ Notably, in NECs and glandular components these mutations often had allele frequencies that implicated their role in early tumor founding clones or their presence in precursor lesions. Moreover, as the glandular tumor components of NECs were morphologically identical to common colorectal adenomas or adenocarcinomas, we propose that NECs evolve from colonic mucosa through the same or a similar malignant transformation process that is well defined as the classical adenoma-carcinoma sequence of the colorectum; ${ }^{23}$ with additional subsequent transdifferentiation into a neuroendocrine cancer phenotype. This hypothesis may apply to most if not all colorectal NECs, as most of these tumors were shown to have at least small neighboring glandular tumor components as putative precursor lesions. ${ }^{9}$ As the genetic basis of colorectal NECs is therefore completely distinct from that reported for NETs or carcinoids, ${ }^{8,10}$ our findings have important implications for tumor classification. If NECs of the colorectum derive from glandular precursors through an adenoma-carcinoma sequence and not from well-differentiated NETs or carcinoids, the current concept of grouping NECs and NETs together in tumor classifications defies their biological basis. ${ }^{3}$ With increasing understanding of the molecular biology of these tumors, a distinct classification may better support accurate characterization and clinical management of these neoplasms in the future. However, as small and large cell NECs showed no obvious genetic differences in our data, the biological basis for these distinct phenotypes remains to be determined.

Colorectal NECs are phenotypically similar to small and large cell neuroendocrine cancers of the lung. In small cell lung cancer, bi-allelic inactivation of TP53 and RB1 is generally found. ${ }^{24}$ In this genetic background, mutational inactivation of NOTCH family genes has been suggested to cause neuroendocrine differentiation. ${ }^{25}$ In our collection of NECs with glandular components, we observed $R B 1$ mutations at high allele frequencies in one case only, whereas a few other cases had lower allele frequencies, indicating mostly subclonal $R B 1$ mutations. In line with this observation, a previous report identified the combination of TP53 and $R B 1$ mutations in only one of six gastrointestinal MANECs. ${ }^{12}$ Moreover, all of the few NOTCH1 gene mutations that we detected were present at low allelic frequencies and therefore also most likely subclonal. Although this implied that in a few colorectal or gastrointestinal NECs neuroendocrine differentiation coincided with TP53 and RB1 mutations, this was uncommon, and suggested a genetic basis distinct from that of small cell lung cancer. Key mutations and molecular drivers of neuroendocrine differentiation in colorectal NECs therefore still remain elusive.

Collectively, we provide additional evidence for a common clonal origin of colorectal NECs and adjacent glandular tumor components. We reveal a mutational spectrum in colorectal NECs that strongly suggests development through the classical adenoma-carcinoma sequence, and demonstrate a genetic basis distinct from colorectal NETs and neuroendocrine lung cancers. Moreover, our data suggest early separation of glandular and neuroendocrine components during malignant transformation of these tumors with subsequent independent mutational evolution.

\section{Acknowledgments}

We are grateful to G Charell and S Sagebiel-Kohler for experimental assistance. Supported by a grant from the Verein zur Förderung von Wissenschaft und Forschung an der Medizinischen Fakultät der LMU München e.V. (VFWF, to DH).

\section{Disclosure/conflict of interest}

The authors declare no conflict of interest.

\section{References}

1 Bernick PE, Klimstra DS, Shia J et al. Neuroendocrine carcinomas of the colon and rectum. Dis Colon Rectum 2004;47:163-169.

2 Lai C, Wang C, Changchien C et al. Neuroendocrine carcinomas of the colon and rectum: result of a 15-year experience. J Soc Colon Rectal Surgeon (Taiwan) 2008;19:87-95.

3 Klimstra DS, Arnold R, Capella C et al. Neuroendocrine neoplasms of the colon and rectum. In: Bosman FT, Carneiro F, Hruban RH et al. (eds). WHO Classification of Tumours of the Digestive System, 4th edn. IARC: Lyon, France, 2010, pp 174-177.

4 Furlan D, Bernasconi B, Uccella S et al. Allelotypes and fluorescence in situ hybridization profiles of poorly differentiated endocrine carcinomas of different sites. Clin Cancer Res 2005;11:1765-1775.

5 La Rosa S, Marando A, Furlan D et al. Colorectal poorly differentiated neuroendocrine carcinomas and mixed adenoneuroendocrine carcinomas: insights into the diagnostic immunophenotype, assessment of methylation profile, and search for prognostic markers. Am J Surg Pathol 2012;36:601-611.

6 Olevian DC, Nikiforova MN, Chiosea S et al. Colorectal poorly differentiated neuroendocrine carcinomas frequently exhibit BRAF mutations and are associated with poor overall survival. Hum Pathol 2016;49: 124-134.

7 Takizawa N, Ohishi Y, Hirahashi M et al. Molecular characteristics of colorectal neuroendocrine carcinoma; similarities with adenocarcinoma rather than neuroendocrine tumor. Hum Pathol 2015;46:1890-1900.

8 Vortmeyer AO, Lubensky IA, Merino MJ et al. Concordance of genetic alterations in poorly differentiated colorectal neuroendocrine carcinomas and associated adenocarcinomas. J Natl Cancer Inst 1997;89:1448-1453.

9 Shia J, Tang LH, Weiser MR et al. Is nonsmall cell type high-grade neuroendocrine carcinoma of the tubular 
gastrointestinal tract a distinct disease entity? Am J Surg Pathol 2008;32:719-731.

10 Ghimenti C, Lonobile A, Campani D et al. Microsatellite instability and allelic losses in neuroendocrine tumors of the gastro-entero-pancreatic system. Int J Oncol 1999;15:361-366.

11 Karkouche R, Bachet JB, Sandrini J et al. Colorectal neuroendocrine carcinomas and adenocarcinomas share oncogenic pathways. A clinico-pathologic study of 12 cases. Eur J Gastroenterol Hepatol 2012;24:1430-1437.

12 Scardoni M, Vittoria E, Volante $\mathrm{M}$ et al. Mixed adenoneuroendocrine carcinomas of the gastrointestinal tract: targeted next-generation sequencing suggests a monoclonal origin of the two components. Neuroendocrinology 2014;100:310-316.

13 Vanacker L, Smeets D, Hoorens A et al. Mixed adenoneuroendocrine carcinoma of the colon: molecular pathogenesis and treatment. Anticancer Res 2014; 34:5517-5521.

14 Kim KM, Kim MJ, Cho BK et al. Genetic evidence for the multi-step progression of mixed glandularneuroendocrine gastric carcinomas. Virchows Arch 2002;440:85-93.

15 Heinemann V, von Weikersthal LF, Decker $\mathrm{T}$ et al. FOLFIRI plus cetuximab versus FOLFIRI plus bevacizumab as first-line treatment for patients with metastatic colorectal cancer (FIRE-3): a randomised, openlabel, phase 3 trial. Lancet Oncol 2014;15:1065-1075.

16 Vosberg S, Herold T, Hartmann L et al. Close correlation of copy number aberrations detected by next-generation sequencing with results from routine cytogenetics in acute myeloid leukemia. Genes Chromosomes Cancer 2016;55:553-567.

17 Jemal A, Siegel R, Xu J et al. Cancer statistics, 2010. CA Cancer J Clin 2010;60:277-300.

18 Neumann M, Vosberg S, Schlee C et al. Mutational spectrum of adult T-ALL. Oncotarget 2015;6:2754-2766.

19 Walter MJ, Shen D, Shao J et al. Clonal diversity of recurrently mutated genes in myelodysplastic syndromes. Leukemia 2013;27:1275-1282.

20 Li Y, Yau A, Schaeffer D et al. Colorectal glandularneuroendocrine mixed tumor: pathologic spectrum and clinical implications. Am J Surg Pathol 2011;35: 413-425.

21 Furlan D, Cerutti R, Genasetti A et al. Microallelotyping defines the monoclonal or the polyclonal origin of mixed and collision endocrine-exocrine tumors of the gut. Lab Invest 2003;83:963-971.

22 Cancer Genome Atlas Network. Comprehensive molecular characterization of human colon and rectal cancer. Nature 2012;487:330-337.

23 Fearon ER, Vogelstein B. A genetic model for colorectal tumorigenesis. Cell 1990;61:759-767.

24 George J, Lim JS, Jang SJ et al. Comprehensive genomic profiles of small cell lung cancer. Nature 2015;524: 47-53.

25 Meder L, Konig K, Ozretic L et al. NOTCH, ASCL1, p53 and $\mathrm{RB}$ alterations define an alternative pathway driving neuroendocrine and small cell lung carcinomas. Int J Cancer 2016;138:927-938.

Supplementary Information accompanies the paper on Modern Pathology website (http://www.nature.com/ modpathol) 